Revue des patrimoines

25 | 2014

Le patrimoine de la Grande Guerre

\title{
La verrière «Aux Morts de la Guerre » de Maurice Denis (1920-1925), de Paris à Montréal
}

\section{Fabienne Stahl and Véronique David}

\section{(2) OpenEdition}

1 Journals

\section{Electronic version}

URL: http://journals.openedition.org/insitu/11359

DOI: 10.4000/insitu.11359

ISSN: 1630-7305

\section{Publisher}

Ministère de la Culture

\section{Electronic reference}

Fabienne Stahl and Véronique David, «La verrière « Aux Morts de la Guerre » de Maurice Denis (1920-1925), de Paris à Montréal », In Situ [Online], 25 | 2014, Online since 12 December 2014, connection on 25 June 2020. URL : http://journals.openedition.org/insitu/11359; DOI : https://doi.org/ 10.4000/insitu. 11359

This text was automatically generated on 25 June 2020 .

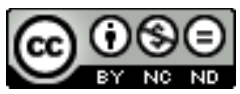

In Situ Revues des patrimoines est mis à disposition selon les termes de la licence Creative Commons Attribution - Pas d'Utilisation Commerciale - Pas de Modification 4.0 International. 


\section{La verrière «Aux Morts de la Guerre » de Maurice Denis (1920-1925), de Paris à Montréal}

Fabienne Stahl and Véronique David

Remerciements : Dominique Bony, Michel Blanc-Garin, Claire Denis, Frédéric Bigo, Marie El Caïdi, l'abbé Thierry de l'Épine, Claire Laville, Benoît Manauté, Gabrielle Montarnal, le père Philippe Ploix, France Trinque.

1 Fin février 2007 était révélée au public canadien, dans l'exposition rétrospective Maurice Denis, le paradis terrestre, une verrière inédite de l'artiste (fig. 1), exécutée en 1925 pour sa présentation dans le Pavillon du vitrail de l'Exposition internationale des arts décoratifs. Le musée des Beaux-Arts de Montréal venait alors de faire l'acquisition de ce vitrail, retrouvé à l'occasion des recherches conjuguées des spécialistes du vitrail du Centre Chastel (Paris) et du Catalogue raisonné de l'œuvre de Maurice Denis (SaintGermain-en-Laye). 
Figure 1

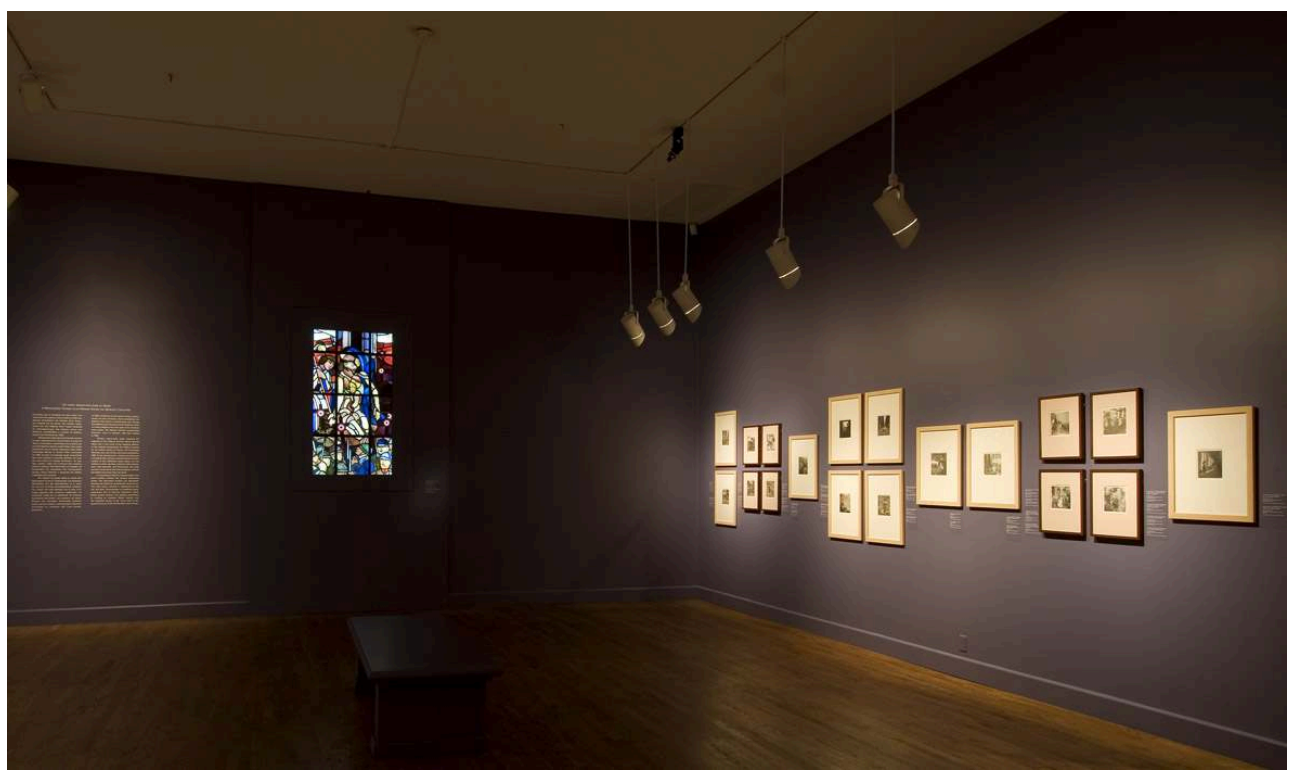

Dernière salle de l'exposition Maurice Denis, le paradis terrestre au musée des Beaux-Arts de Montréal (Canada), 22 février-20 mai 2007.

(c) Musée des Beaux-Arts de Montréal.

Les circonstances de cette découverte méritent d'être relatées, cette verrière ayant une histoire singulière, qu'il nous a semblé important d'éclaircir pour corriger les erreurs publiées, en particulier relativement aux nombreux travaux préparatoires longtemps attribués à tort à l'église de Gagny ${ }^{1}$. Or, la commande d'un monument dédié aux morts de la paroisse pour l'église Saint-Germain de Gagny, passée à l'automne 1919, est pourtant sans rapport avec celle de l'église Saint-Roch de Paris. À Gagny, les interlocuteurs du peintre sont l'abbé Desgroux, ancien vicaire de Saint-Germain-enLaye, et l'architecte Georges Brochard, qui envisagent la réalisation d'une peinture murale - jamais d'un vitrail! - de forme semi-circulaire, dans la chapelle SainteJeanne-d'Arc, dite « chapelle du Souvenir » (anciennement chapelle Sainte-Geneviève) (fig. 2). 
Figure 2

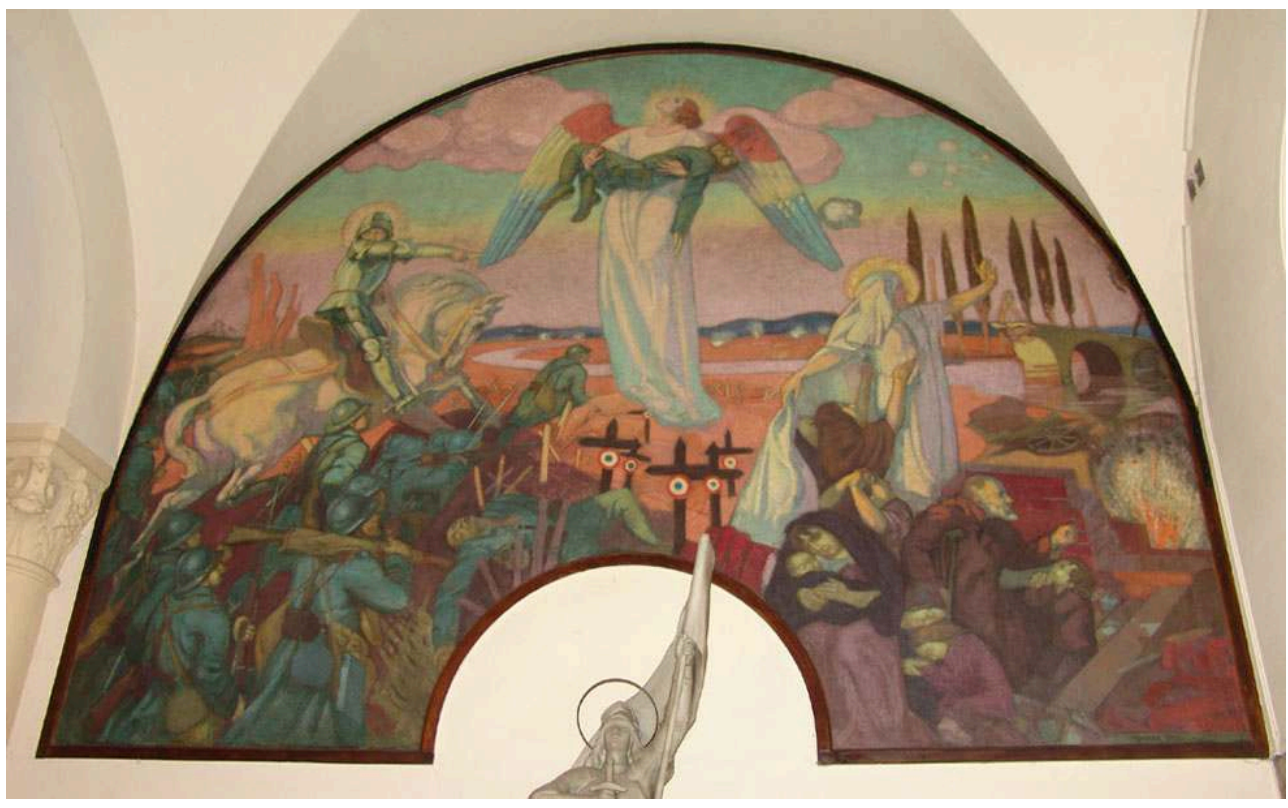

Maurice Denis, In memoriam. Aux morts de la paroisse de Gagny ou [La Bataille de la Marne], 1920, huile sur toile marouflée, $390 \times 387 \mathrm{~cm}$.

(c) Fabienne Stahl, Catalogue raisonné Maurice Denis.

3 L'historique de la commande pour Saint-Roch de Paris a pu être restitué grâce à la découverte de correspondances inédites, échangées entre l'artiste et ses commanditaires $^{2}$; cette documentation archivistique et graphique exceptionnelle est conservée, pour l'essentiel, au musée départemental Maurice Denis de Saint-Germainen-Laye et aux archives de l'archevêché de Paris.

\section{Acte 1}

\section{Historique de la commande et travaux préparatoires}

4 L'église Saint-Roch, située 284, rue Saint-Honoré dans le premier arrondissement de Paris, est l'une des plus vastes de la capitale. Cet édifice phare, dont l'architecture date de la fin XVII -début $\mathrm{XVIII}^{\mathrm{e}}$, a été classé au titre des Monuments historiques le 7 décembre 1914. Elle est considérée comme la « paroisse des artistes » - par allusion au nombre d'entre eux qui y ont été inhumés ou dont on y a célébré les obsèques (André Le Nôtre, Pierre Corneille, Denis Diderot, Jean Honoré Fragonard...) - mais surtout en raison de la riche collection d'œuvres d'art qu'elle conserve en son sein ${ }^{3}$.

5 La commande à Maurice Denis, célèbre pour son investissement dans le renouveau de l'art religieux, d'une verrière dédiée aux morts de la paroisse s'inscrit dans un vaste mouvement de commémorations tant dans le domaine civil que religieux suscité par la Grande Guerre. 
Figure 3

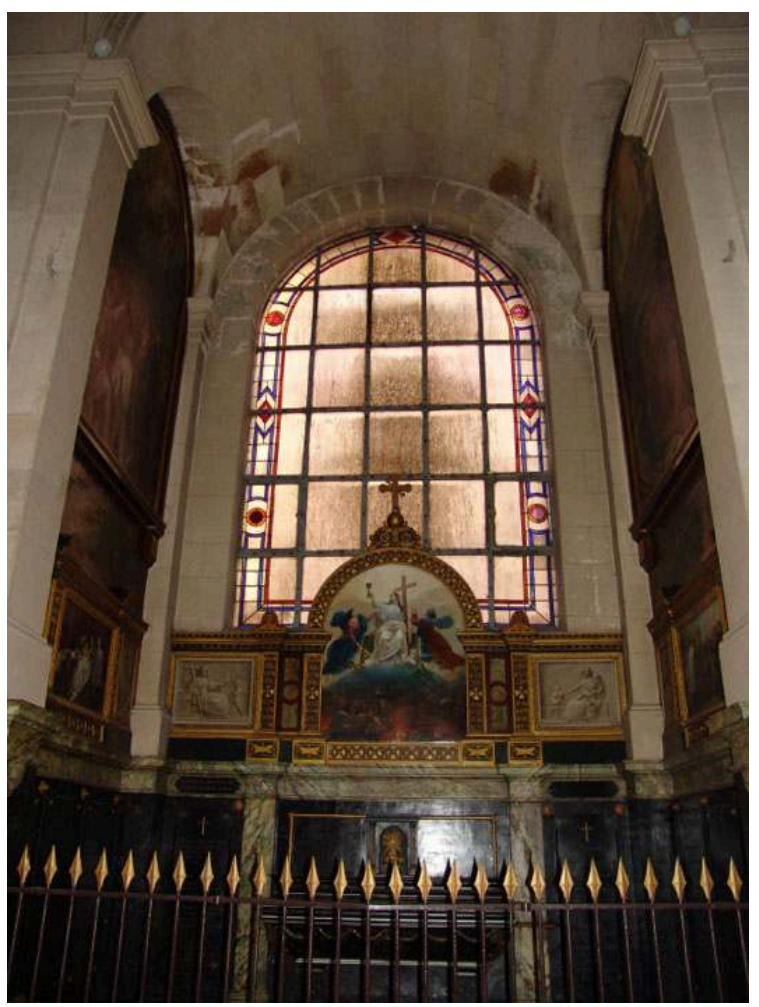

La chapelle du Purgatoire de l'église Saint-Roch de Paris (photographie contemporaine).

(c) Fabienne Stahl, Catalogue raisonné Maurice Denis.

6 La première mention de cette commande est connue par une lettre du curé Peuportier, curé de la paroisse, datée du 27 janvier 1920, où il remercie Denis de bien vouloir accepter sa demande: "J'ai l'honneur de vous adresser comme je l'ai promis, les dimensions de la baie de la chapelle des âmes du Purgatoire (fig. 3) pour laquelle vous voulez bien préparer les cartons d'un vitrail en souvenir de la grande guerre : Hauteur 5,03 L $3,22^{4}$ ». À même cette lettre, Denis croque une première idée (fig. 4), qu'il transpose rapidement en esquisse sur calque (fig. 5), pour la transmettre au curé pour validation. Ce dernier lui donne un accord de principe, avec quelques réserves :

Je suis bien en retard pour répondre à votre lettre du deux courant. Le motif en est que je voulais montrer votre calque au conseil et au comité paroissial afin d'avoir leur avis et de vous le transmettre. Je suis heureux de vous informer que tous goûtent fort votre projet et estiment que son exécution produira un bel effet. Une simple petite objection qui n'en est pas une parce que vous l'auriez résolu vousmême en travaillant votre projet est que le poids du corps de Notre Seigneur, mort, doit être bien lourd, dans la pose que vous lui assignez et par conséquent trop lourd pour que la Vierge, si épuisée de douleurs, puisse le soutenir. J'espère qu'il vous sera possible de préparer votre maquette à temps pour qu'on puisse l'exposer le 27 mars à cause des nombreuses visites faites à Saint Roch pendant la semaine sainte $^{5}$. 
Figure 4

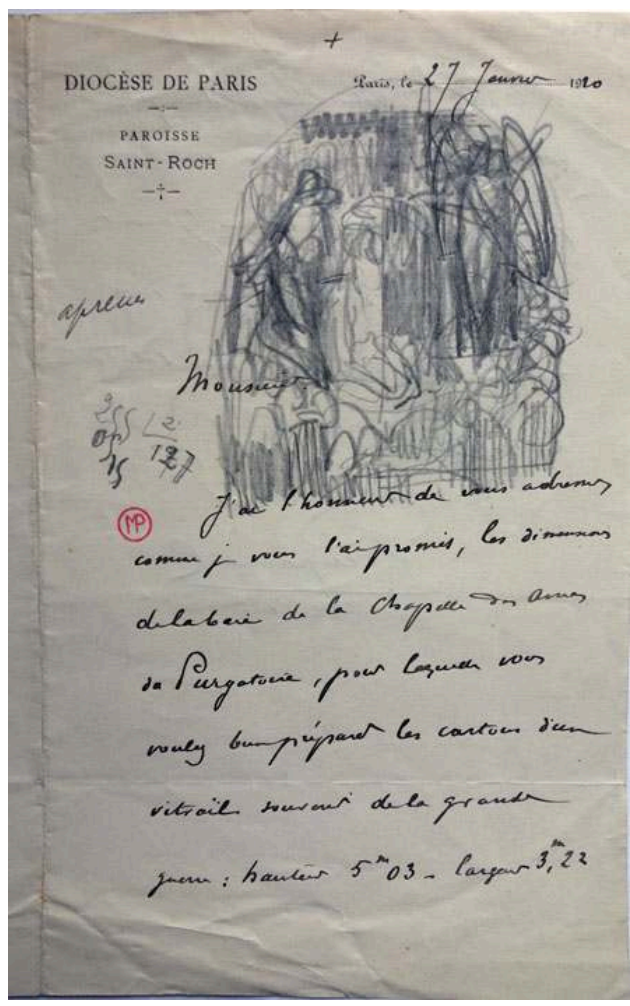

Lettre de l'abbé Peuportier, curé de la paroisse Saint-Roch de Paris, 27 janvier 1920, avec le premier croquis de l'artiste pour le projet de verrière (Documentation du musée départemental Maurice Denis, Saint-Germain-en-Laye, donation de la famille Denis, inv. Ms 8532).

(c) Fabienne Stahl, Catalogue raisonné Maurice Denis. 
Figure 5

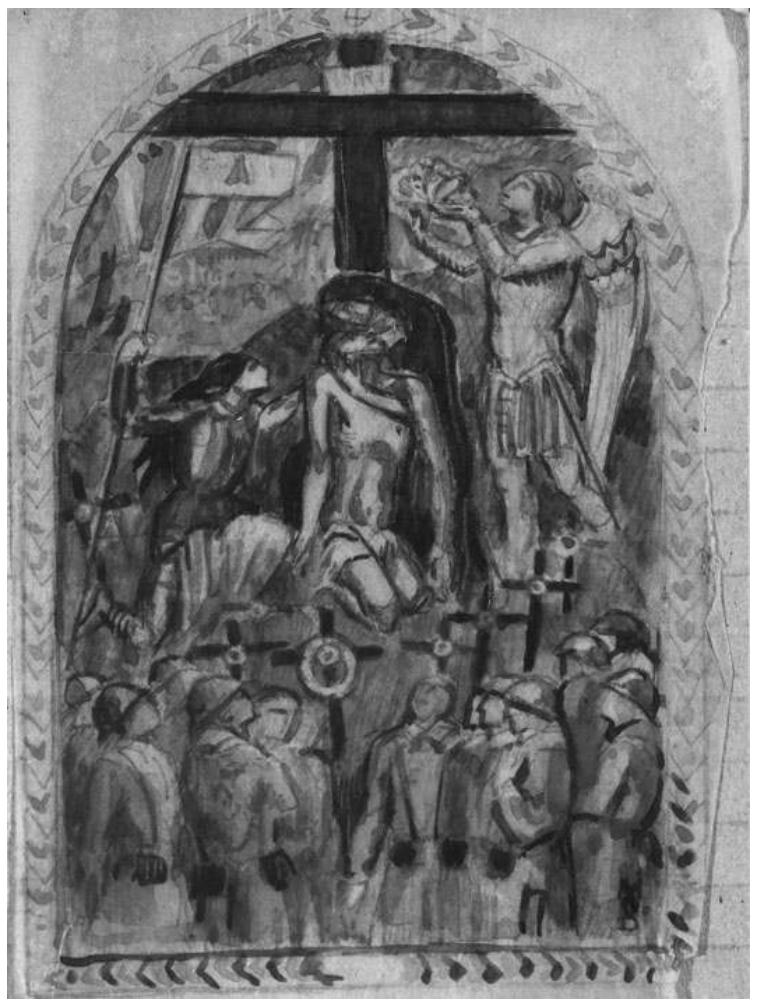

Maurice Denis, Pietà (première esquisse pour le vitrail de l'église Saint-Roch de Paris), crayon et gouache sur papier calque, collé sur papier, $14,5 \times 10$ cm, vendu à Tokyo, 1983 (non localisé depuis).

(c) Fabienne Stahl, Catalogue raisonné Maurice Denis.

7 Au début de l'été 1920, Denis présente un devis, après consultation du verrier, comme indiqué au curé :

D'après la lettre que j'ai de M. Gsell Maury, peintre verrier, je puis fixer le prix du vitrail (y compris la pose, mais non compris la dépose des vitres actuelles ni la serrurerie, s'il est nécessaire de renouveler les fers), au chiffre global de $24.000 \mathrm{fr}$. Vous m'aviez demandé de vous renseigner à ce sujet. Voilà qui est fait. Il est bien entendu que je prends l'engagement d'exécuter un carton de grandeur, d'en surveiller l'exécution en matière choisie et de collaborer avec Gsell Maury à la peinture sur verre. L'invention du dessin, l'exécution et la mise en place du vitrail sont donc compris dans le chiffre ci-dessus; et vous n'avez pas d'autre frais à prévoir sauf ceux mentionnés plus haut (dépose et fers) ${ }^{6}$.

8 Après quoi, la paroisse tarde à se décider. Le curé demande à Denis de patienter un peu pour avoir la décision du conseil et sans doute trouver les financements ${ }^{7}$. 
Figure 6

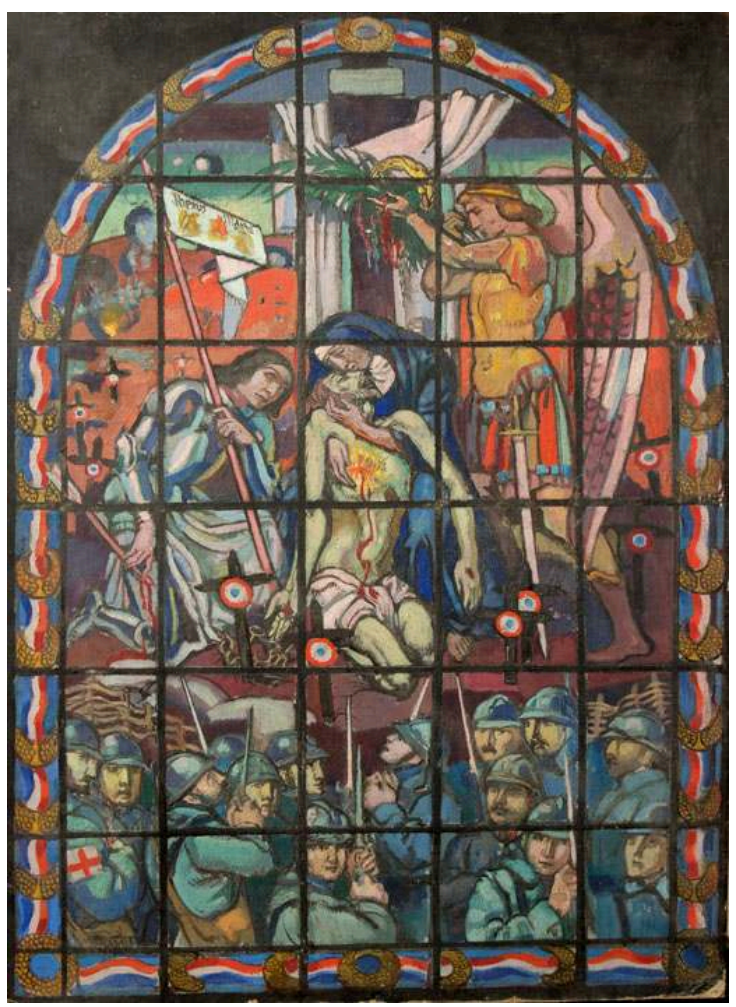

Maurice Denis, Aux morts de la guerre, première maquette pour le vitrail de l'église Saint-Roch de Paris, vers 1920, huile sur carton, $72 \times 52,5 \mathrm{~cm}$, coll. part.

(c) Olivier Goulet, Catalogue raisonné Maurice Denis.

9 À l'automne 1920, le projet est toujours en "suspens $»^{8}$ et Denis souhaite présenter son esquisse dans l'Exposition d'art chrétien moderne au Pavillon de Marsan'. Comment se présente cette composition (fig. 6) ? Au centre, le Christ descendu de la croix, au cœur rayonnant, fait référence au Christ du Sacré-Cœur. Il est soutenu par sa mère, dans un geste fusionnel et douloureux, qui rappelle les souffrances des endeuillés de la guerre. Les personnages siègent à même le champ de bataille, semé de croix portant la cocarde des soldats morts au champ d'honneur. La composition est centrée sur une Pietà - qui reprend celle du projet de monument aux morts pour l'église Sainte-Marguerite du Vésinet ${ }^{10}$, peint en 1919 (fig. 7) (et repris en lithographie), pour lequel il avait fait poser une femme pour la figure du Christ! (fig. 8) - entourée des deux saints protecteurs de la France, sainte Jeanne d'Arc (qui vient d'être canonisée en 1920) et saint Michel archange. Une des nouveautés réside dans l'association de la Pietà au Christ du SacréCœur. L'iconographie originale de cette Pietà illustre bien les idées alors défendues par Maurice Denis, qui cherche à émouvoir le fidèle par l'actualisation des scènes sacrées. Au premier plan, séparés du monde divin par une clôture de branchage, les poilus dans la tranchée sont représentés d'une manière réaliste, en uniforme bleu horizon, casqués, les fusils Lebel dressés et la musette en bandoulière. Certains soldats - certains visages indiquent qu'il pourrait s'agir de portraits - tournent le regard vers le spectateur, comme pour le conduire à entrer dans l'image. À l'arrière-plan à gauche, un obus explose au milieu des soldats dans la tranchée. Une bordure de drapeaux français et de couronnes de lauriers unit les deux registres. 
Figure 7

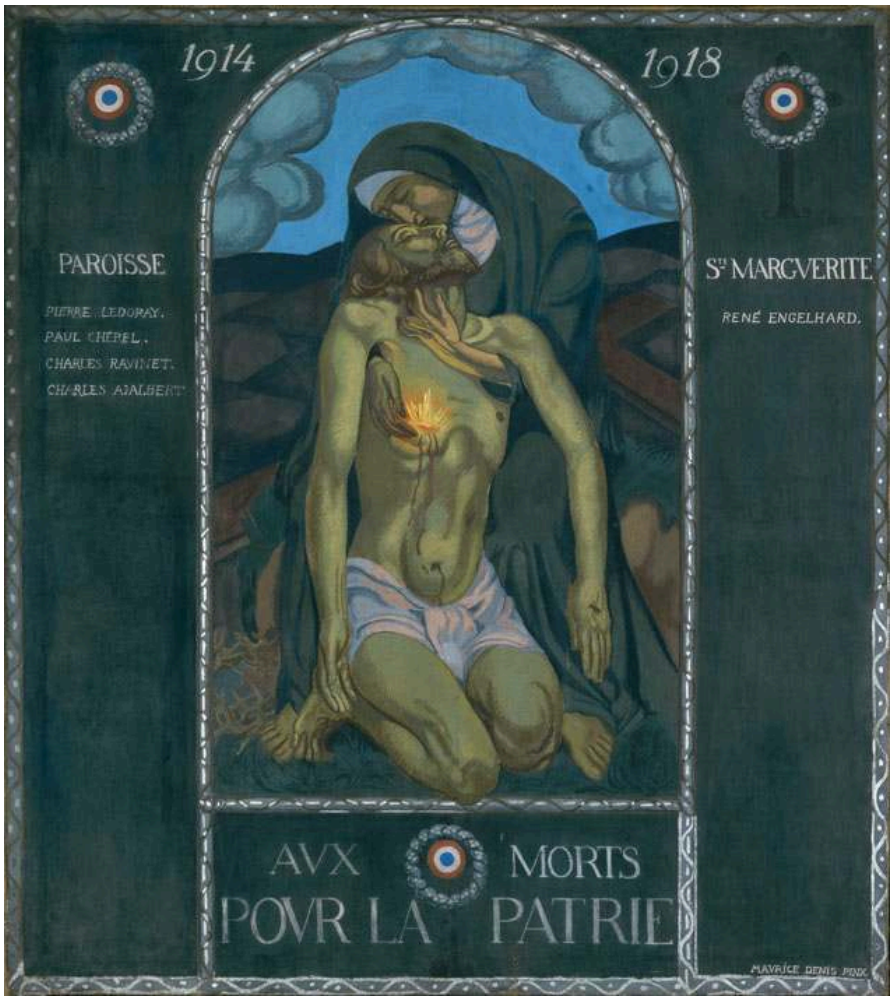

Maurice Denis, Aux morts pour la patrie, projet pour le monument aux morts de l'église SainteMarguerite du Vésinet, vers 1919, huile sur toile, $180 \times 150 \mathrm{~cm}$, coll. part.

(c) Sully-Jaulmes, Catalogue raisonné Maurice Denis. 
Figure 8

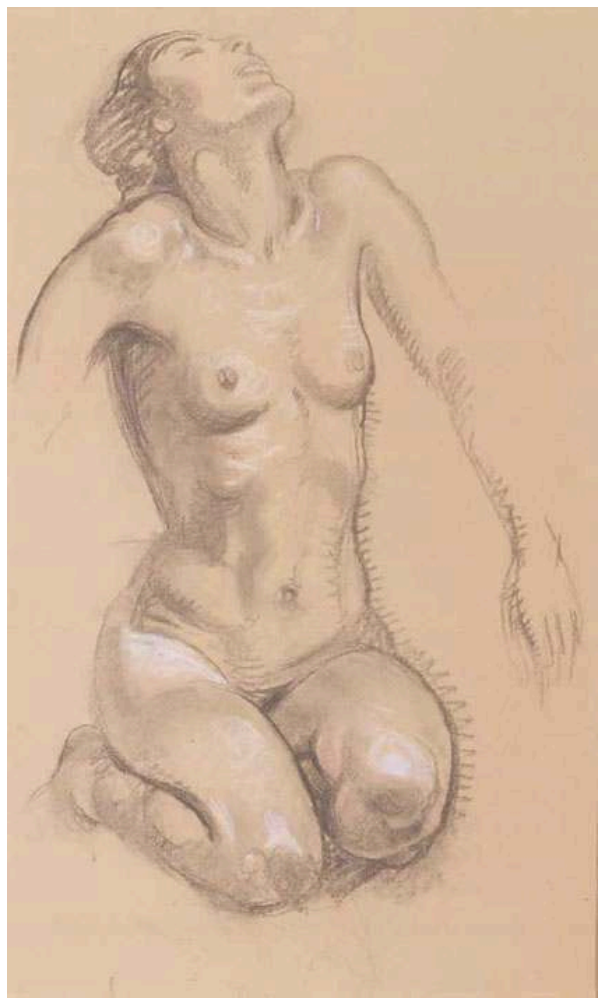

Maurice Denis, Femme nue à genoux, étude de modèle féminin pour le Christ mort, vers 1919, 52,5 x 35 cm, Musée municipal de Saint-Germain-en-Laye, inv. 944.3.1.

(c) Musée municipal de Saint-Germain-en-Laye.

10 Au printemps suivant, au retour de l'exposition, l'esquisse est exposée dans l'église, sans doute pour recueillir l'intérêt des paroissiens ${ }^{11}$. À cette occasion, Denis reçoit le témoignage de l'un d'eux, assez touchant, qu'il nous plait à citer ici dans son intégralité :

Jusqu' à ce jour, je vous ignorais ou presque. Tout à l'heure, à Saint-Roch, ma paroisse, j'ai vu un projet de vitrail où j'ai lu votre nom et où vous avez rendu sensible le sacrifice de nos chers morts de la guerre en le rapprochant du sacrifice du Christ Jésus... Je suis un ignorant et vous êtes un maître, me dit-on ; pardonnezmoi mais vous m'avez ému jusqu'aux larmes et j'ai besoin de vous le dire. Qu'il est profond et expressif le langage que vous parlez... Ah! il m'est bien égal, à moi, que certains visages soient verts : mon âme y trouve son compte, et c'est la première fois qu'un tableau religieux parle à mon âme. Soyez en remercié au fond du cœur. Et je ne vous parlerai pas de mon "admiration» ni ne vous prodiguerai les termes emphatiques que vous ne connaissez que trop sans doute : en ce mot merci je mets tout ce que j'ai sur le cœur !

Si j'osais, je vous demanderais en toute simplicité si vous avez mieux fait ailleurs et où ? pour y aller pieusement... Ayez la charité de me pardonner. En ce jour de fête je prie Dieu qu'Il vous permette de multiplier longuement parmi nous de pareilles leçons et donne à un plus grand nombre la grâce d'en saisir le sens profond. Merci ${ }^{12}$.

11 Après cette longue présentation auprès des paroissiens, le curé Peuportier se décide à réécrire à Denis pour lui faire part des suites, plutôt défavorables, du projet :

Je me permets aujourd'hui de vous demander, si cela vous est possible de nous fournir une nouvelle esquisse en vous donnant seulement cette indication qu'au bas du sujet que vous avez choisi figurent quelques soldats pour donner satisfaction à plusieurs paroissiens pour lesquels ce serait une consolation de retrouver sur le 
vitrail les traits de celui qui est mort pour la France sous le regard du Christ. Si vous désiriez me voir et causer avec quelques uns que ce monument intéresse davantage nous nous tiendrons à votre disposition, à mon presbytère de Saint-Roch au jour et à l'heure que vous désigneriez ${ }^{13}$.

Figure 9

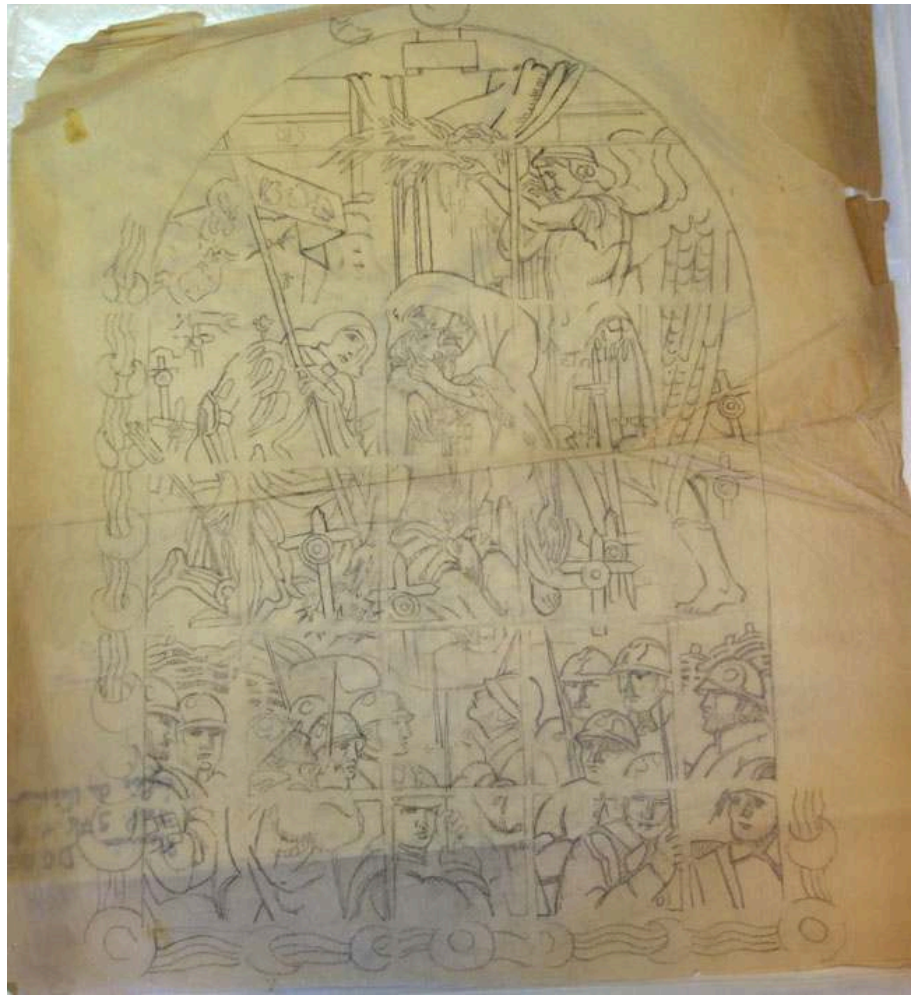

Maurice Denis, Aux morts de la guerre, étude sur calque, 72,5 x 63,5 cm, musée départemental Maurice Denis, Saint-Germain-en-Laye, inv. PMD 976.1.1361.

(c) Fabienne Stahl, Catalogue raisonné Maurice Denis.

Les choses restent ainsi... jusqu'à l'arrivée en octobre d'un nouveau curé, Henri Victor Couget (qui y restera jusqu'en 1941). C'est sans doute à cette époque (en avril 1922), que Denis réalise, à partir d'un calque (fig. 9), une nouvelle esquisse en camaïeu (fig. 10), légèrement modifiée ${ }^{14}$ et qu'il récupère la première ${ }^{15}$. Au printemps 1922, on pourrait penser le projet acquis, à la lecture de la lettre que le curé adresse à Denis :

À la date convenue, je m'empresse de vous dire que nous acceptons le nouveau projet de vitrail, dont vous avez eu l'obligeance de me donner une nouvelle esquisse; quelques personnes ont seulement exprimé le désir que fut réduit le nombre des croix de bois sur les tombes.

Il serait bien à souhaiter que le vitrail fut terminé et placé en novembre prochain, ainsi que les plaques de marbre sur lesquelles seront inscrits les noms des soldats défunts. Pour ces plaques de marbre je n'ai pas d'idée précise; mais je crois qu'il faudrait s'inspirer des autres plaques déjà placées dans l'église, comme celle de Corneille, par exemple.

Vous m'avez dit que vous estimiez la dépense de cet ensemble, vitrail et plaques à environ 30000 francs ; j'espère que ce prix ne sera guère dépassé16. 
Figure 10

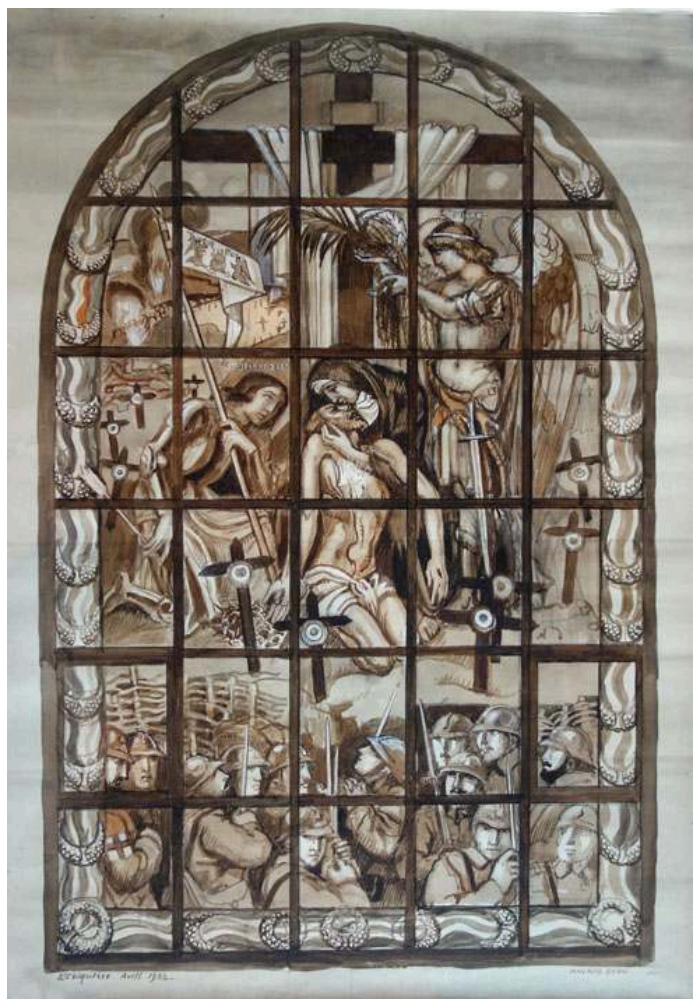

Maurice Denis, Aux morts de la guerre, maquette, gouache sur papier, 87 x $61 \mathrm{~cm}$, daté B.G. : $2^{\mathrm{e}}$ esquisse avril 1922, musée départemental Maurice Denis, Saint-Germain-en-Laye, inv. PMD 976.1.694. (c) Fabienne Stahl, Catalogue raisonné Maurice Denis.

13 Fin mai 1922, il est envisagé de retirer le retable présent dans la chapelle pour installer le vitrail ${ }^{17}$. Denis craint que celui-ci ne puisse être achevé pour le mois de novembre ${ }^{18}$, mais récupère son esquisse en vue de la faire agrandir ${ }^{19}$. Pourtant, arrivent de nouvelles complications ${ }^{20}$... Le financement semble problématique, ce que confirme une lettre du vicaire général Thomas adressée au curé ${ }^{21}$. L'improbable arrive : le projet est tant révisé à la baisse que le vitrail est supprimé, le monument commémoratif se limitant à la pose de plaques mentionnant les noms des morts ${ }^{22}$. Denis ne peut que souscrire à la décision, comme il l'écrit à son gendre Marcel Poncet:

Mon affaire de St Roch est décidément ratée, le curé après m'avoir écrit de commencer a dû reculer, devant l'opposition irréductible du conseil de fabrique, et je lui ai rendu sa parole... Voilà où on en est à Paris, mon projet est trop révolutionnaire! ${ }^{23}$

\section{Acte 2}

\section{La reprise du projet pour l'exposition de 1925}




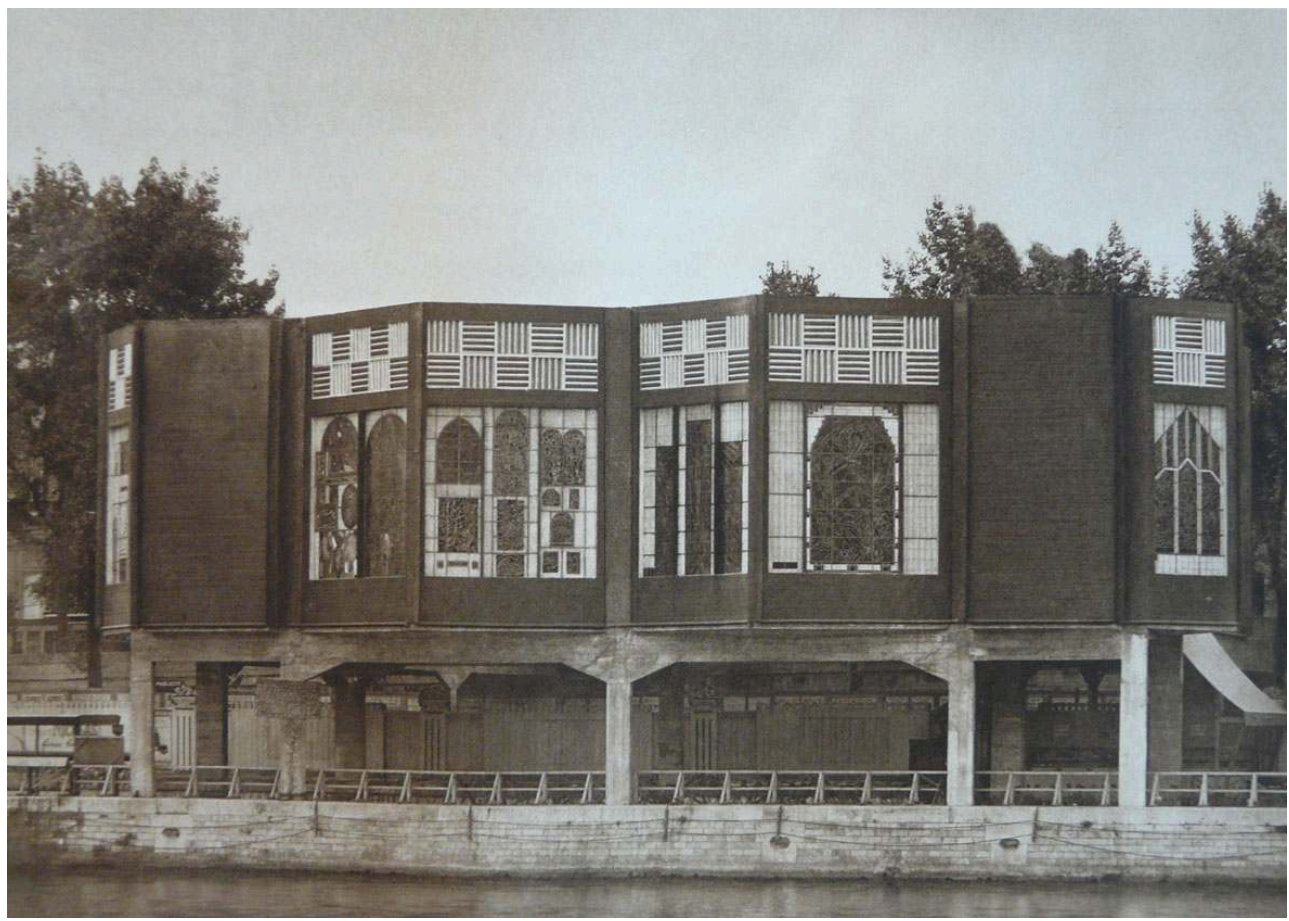

Vue extérieure du Pavillon du vitrail de l'Exposition internationale de 1925.

(c) Benoît Manauté

L'affaire aurait pu en rester là, Maurice Denis conservant ses maquettes dans son atelier. Pourtant, trois ans plus tard, on retrouve la partie centrale de son projet traduite en vitrail dans le Pavillon du vitrail de l'Exposition internationale des Arts décoratifs et industriels modernes de 1925 (aucun carton à grandeur d'exécution n'a été retrouvé). Ce bâtiment, construit par l'architecte Lucien Woog, a la forme curieuse d'un paravent translucide éployé en bordure de la Seine (fig. 11). Il relève de la classe 6 " Art et industrie du verre » présidée par Jacques Gruber ${ }^{24}$. Trente-sept peintres verriers, en majorité parisiens, y présentent cent dix verrières d'une grande hétérogénéité présentées dans vingt «baies». Le vitrail de Maurice Denis fait partie des œuvres présentées par l'atelier Hébert-Stevens et Rinuy dans la baie IX sous le titre Pietà. Une photographie en noir et blanc présente l'ensemble des vitraux de l'atelier HébertStevens et Rinuy (fig. 12) : document précieux, parce que seul témoignage connu d'une «baie » complète dans ce Pavillon du vitrail. Cette prise de vue a été faite à la demande de Chirol pour la préparation de son article dans La Vie catholique du 22 août 1925 et lui a été envoyée par l'atelier le 11 juillet 1925, avec la liste des vitraux, intéressante à transcrire ici :

1. Guérison de Marie Lemarchand (Lourdes) carton de P. Couturier

2. Vierge par André Rinuy

3. Vierge par Hébert Stevens

4. N.D des Prairies, carton de $\mathrm{M}^{\mathrm{me}}$ P. Peugniez

5. Saint François d'Assise, carton de André Lecoutey

6. Soir d'Emmaüs, carton de Georges Gallet

7. Le Couronnement de la Sainte Vierge, en dessous l'Annonciation, Nativité et Christ Vierge au Calvaire par André Rinuy

8. Pietà, carton de Maurice Denis

9. Vierge consolatrice et Saint Michel, carton de G. Desvallières 
10. La petite Sœur Thérèse et l'Enfant Jésus par Hébert Stevens

11. Noli me tangere par Hébert Stevens ${ }^{25}$

Figure 12

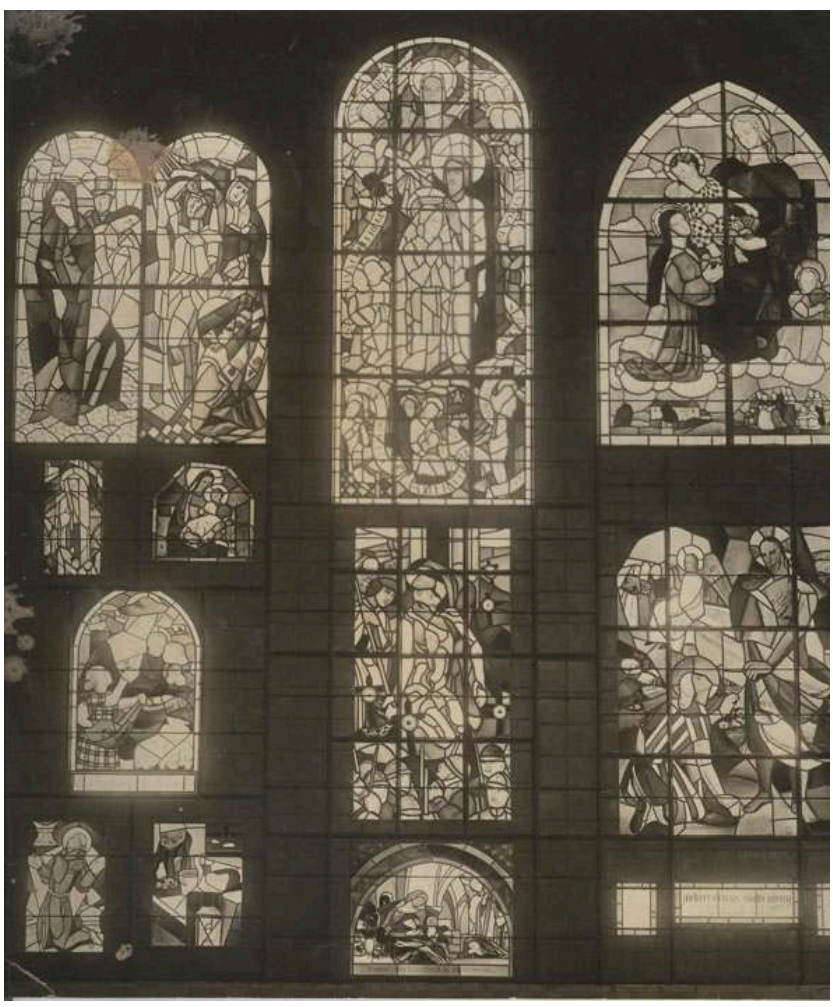

Vue intérieure du Pavillon du vitrail de l'Exposition internationale de 1925, la baie Jean HébertStevens/Rinuy, photographie dans le fonds d'atelier Jean Hébert-Stevens.

(c) Véronique David.

Dans le Pavillon du vitrail, il est à remarquer que l'iconographie du souvenir est peu présente alors même qu'elle connaît un développement très important dans les édifices de la Reconstruction. La seule verrière au thème directement commémoratif est celle de Maurice Denis. On peut y ajouter celle présentée par Jean Hébert-Stevens, La Vierge consolatrice et saint Michel d'après un carton de George Desvallières, ainsi que la Légende du pays chartrain de Charles Lorin qui porte l'inscription de dédicace à son fils Stéphane victime de la Grande Guerre le 15 octobre 1917.

16 Mais regardons précisément comment la maquette de Denis a été traduite en vitrail (fig. 13). 
Figure 13

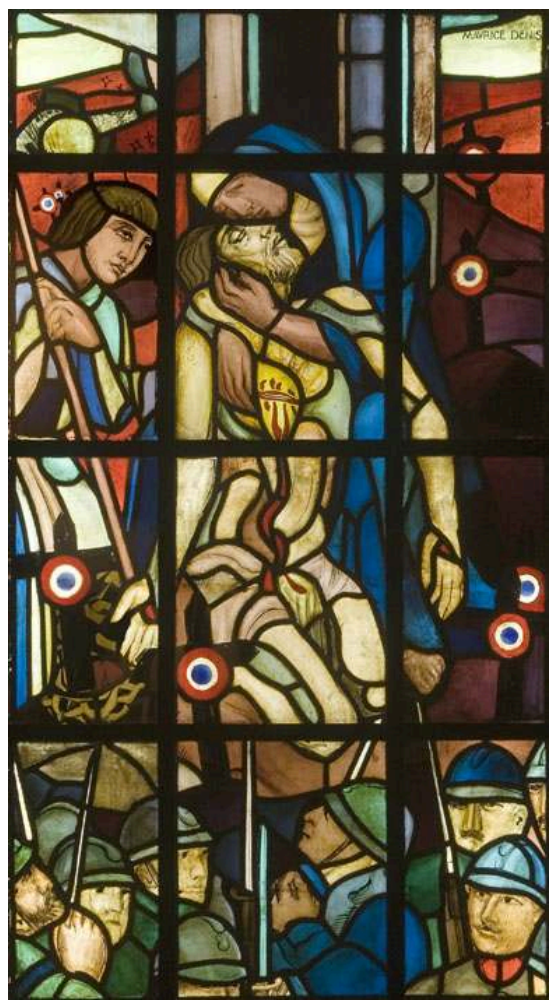

Jean Hébert-Stevens et Albert Rinuy, Pietà, d'après Maurice Denis, présentée dans le Pavillon du vitrail de l'Exposition internationale de 1925, vitrail, 171 x 109,5 cm, musée des Beaux-Arts de Montréal, inv. 2006.83.

(c) Musée des Beaux-Arts de Montréal.

17 Au lieu des trente panneaux prévus pour la partie centrale à laquelle s'ajoutaient vingtdeux panneaux de bordure, le vitrail définitif est réduit à douze panneaux, découpés selon le principe de barlotières orthogonales proposé par Denis dans sa maquette. Le cadrage beaucoup plus resserré concentre l'attention sur les acteurs principaux de la tragédie. Saint Michel est supprimé et remplacé par des croix de cimetière militaire. De l'étendard de sainte Jeanne d'Arc ne subsiste que la hampe. La croix du Christ n'est visible que dans sa partie inférieure. Le nombre de figures des poilus est réduit à neuf, au lieu des quatorze à l'origine. Les bordures sont supprimées.

Pourquoi cette réduction? L'espace dévolu à l'atelier Jean Hébert-Stevens était naturellement non extensible et le vitrail de Denis devait laisser la place aux autres œuvres.

19 La traduction de la maquette en vitrail est confiée à Albert Rinuy, de l'atelier HébertStevens. Cette opération est particulièrement délicate car le passage de la maquette, c'est-à-dire d'un support opaque à un matériau transparent, le verre, conduit nécessairement à des transformations profondes. Ainsi, si la composition du vitrail reprend bien celle de la maquette, son effet est très différent de l'œuvre initiale du fait du matériau même du verre, de son morcellement, et des techniques de traitement du verre par la peinture et la gravure. La complicité de l'artiste et du peintre verrier est donc capitale dans ce travail.

La coloration dense est intensifiée par la qualité des verres antiques utilisés. La palette est dominée par des tonalités bleues et rouges qui ne sont pas sans rappeler la grande 
tradition du vitrail médiéval, symbole absolu pour Maurice Denis du génie français, qu'il vantera dans son Histoire de l'art religieux parue en 1939. La présence également assez importante de blanc pourrait être lue comme une allusion aux couleurs du drapeau national.

Une utilisation habile des procédés techniques de gravure attire l'attention sur les motifs du cœur du Christ gravé sur du verre rouge et teint au jaune d'argent et sur les cocardes gravées sur verre rouge et peintes à l'émail bleu dans la partie centrale ${ }^{26}$. L'idée sous-jacente est la mise en parallèle du sacrifice du Christ pour l'humanité et de celui des soldats pour la patrie.

Les procédés d'application de peinture sont classiques. Les visages sont rendus à partir d'un jus de grisaille qui en couvre toute la surface, le travail du pinceau marquant les traits des figures parfois renforcés par un lavis et éclaircis par des enlevés. Ces soldats évoquent la robustesse des personnages de Marcel Gromaire.

La mise en plomb adopte deux partis : soit le respect du dessin prédomine - c'est le cas pour les poilus dont les plombs soulignent précisément les contours des visages et des casques, soit le morcellement prend le dessus ; ainsi, pour le corps du Christ découpé dans un très grand nombre de pièces de verre, sans lien avec les nécessités techniques (solidité du verre qui ne peut dépasser une certaine surface sans être compromise). Le choix de ce parti ne peut être imputable au seul peintre verrier, Maurice Denis ayant pour habitude de suivre de très près toutes les étapes de la fabrication; en cela, il suit la tradition du vitrail médiéval qui refuse de réduire le vitrail à un tableau sur verre traité selon les codes académiques de la perspective et du modelé.

La réalisation de ce vitrail ne semble pas avoir satisfait Maurice Denis. En témoigne la lettre de Rinuy à Denis le 2 juillet 1925 : «J'ai patiné la Pietà de mon mieux comme vous me le demandiez. Mais combien j'aurais préféré réussir le vitrail. Je suis tout à votre disposition pour le patiner d'avantage si vous le jugez utile. ${ }^{27}$ » Rinuy exprime son regret de ne pas lui avoir donné satisfaction et son désir de se rattraper en appliquant davantage de patine sur le vitrail. En effet, la patine, posée au revers du vitrail, sert à réguler la lumière, l'adoucir. Soit elle est appliquée au départ, soit, comme dans ce casci, après coup, comme une sorte de retouche. La maladresse de Rinuy s'explique aussi par la jeunesse de l'atelier, créé seulement une année auparavant.

La reprise par Denis de sa maquette relève d'une pratique habituelle aussi bien de la part des artistes que des peintres-verriers créateurs. Il est logique de vouloir "rentabiliser » un projet qui a exigé un investissement important. Le choix de cet atelier s'explique aisément dans ce cas. Jean Hébert-Stevens, sa femme Pauline Peugniez et André Rinuy, son collaborateur, étaient bien connus de Denis. Ils étaient entrés en 1920 aux Ateliers d'art sacré, un an après leur fondation par Maurice Denis et George Desvallières, et avaient fondé en 1924 un atelier de vitrail (où André Rinuy ne reste que jusqu'en 1939) à Paris. Cet atelier qui se veut un lieu de rencontre entre artistes et peintres-verriers joue un rôle fondamental dans l'ouverture de l'art sacré à la modernité. Partisan du rapprochement de l'art du vitrail et de la peinture, l'atelier réalise les cartons de nombreux peintres. À l'Exposition internationale de Paris en 1925, outre ceux destinés au Pavillon du vitrail, il réalise ceux de Denis, Desvallières et du père Couturier pour l'église du Village français. Par ailleurs, l'atelier réalisera celui de Denis pour l'église de Margny-aux-Cerises (Oise, 1930), ceux de George Desvallières pour la chapelle de l'Ossuaire de Douaumont (Meuse, 1927), ceux de Valentine Reyre pour l'église de l'Immaculée Conception à Audincourt (Doubs, 1927-1932) et pour 
l'église Saint-Martin de Vendhuile (Aisne, 1928-1932), ceux du père Couturier pour le couvent Sainte-Sabine à Rome (Italie, 1937), ou encore ceux de Boris Mestchersky pour l'église de Plaine (Bas-Rhin, 1935).

Au fil des années, Jean Hébert-Stevens cumulera bien d'autres activités : sociétaire de la Société nationale du Salon d'Automne, il fait partie du comité de rédaction de la revue L'Art sacré de 1935 à 1939. En 1937, il est membre du jury du Pavillon du vitrail de l'Exposition internationale de Paris et participe au programme de douze verrières destinées, après leur exposition au Pavillon pontifical, à être intégrées à la cathédrale Notre-Dame de Paris (projet abandonné). Ses créations et celles de sa femme prennent place dans de nombreuses églises, notamment dans celles de la Reconstruction dues aux architectes Charles Duval et Emmanuel Gonse en Picardie. En 1939, il organise avec Pauline Peugniez et le père Couturier l'exposition Vitraux et tapisseries modernes au Petit-Palais qui représente un véritable jalon dans l'histoire du vitrail du $\mathrm{xx}^{\mathrm{e}}$ siècle grâce à la commande de cartons de vitraux à trois grands peintres contemporains dont il s'agit de la première intervention dans ce domaine, Jean Bazaine, Marcel Gromaire et Georges Rouault. L'histoire de l'atelier se poursuit après la Seconde Guerre mondiale avec Paul Bony (1911-1982) rentré à l'atelier en 1934 qui épouse en 1943 leur fille Adeline Hébert-Stevens (1917-1998), également peintre-verrier.

\section{Épilogue}

\section{Paris / Montréal : de la redécouverte à la mise au jour}

Été 2005 : nous venons à la rencontre de Dominique Bony dans l'atelier de la rue Ferrandi pour examiner la verrière du curé d'Ars de Maurice Denis, issue de l'église du Village français dans l'Exposition des arts décoratifs 1925 - la sœur de cette verrière, Sainte Marguerite Marie Alacoque, a alors déjà rejoint les collections du musée départemental de l'Oise à Beauvais. Nous rêvons à une issue muséale pour cette figure, qui rejoindra effectivement le musée des Beaux-Arts de Reims peu de temps après. Sous la conduite bienveillante de notre hôte, nous sortons différents panneaux, en évoquant les questions de sauvegarde et d'études de ce merveilleux fonds. Nous programmons d'autres rencontres du même type pour l'automne.

\section{Retour aux sources et à la bibliographie}

Si dans son étude détaillée du fonds d'atelier de Jean Hébert-Stevens / Rinuy, Florence Ledoux avait bien repéré la verrière qui nous occupe, elle l'avait reproduite sans l'avoir identifiée ${ }^{28}$. Le rapprochement entre la maquette de Denis et cette photographie nous a engagées à mener plus avant notre enquête dans le fonds d'atelier de Dominique Bony, petit-fils de Jean Hébert-Stevens... Une fois un premier panneau avec la signature «MAVRICE DENIS» localisé, il ne nous reste plus qu'à persévérer dans nos investigations pour localiser les autres pièces du puzzle! Séance de prises de vue improvisée des différents panneaux, suivie d'un montage sur Photoshop qui redonne vie à cette verrière.

Nathalie Bondil, conservatrice du musée des Beaux-Arts de Montréal, est alors en train de faire sa sélection d'œuvres pour la section « arts décoratifs » qu'elle souhaite ajouter dans la rétrospective Maurice Denis conçue par le musée d'Orsay. Coup de foudre pour 
ce vitrail inédit, et engagement de la restauration en vue d'une présentation dans l'exposition.

30 La restauration est confiée à Michel Blanc-Garin, qui œuvre sur notre verrière durant l'automne 2006 dans son atelier de Bagnolet (Clair Vitrail).

Figure 14

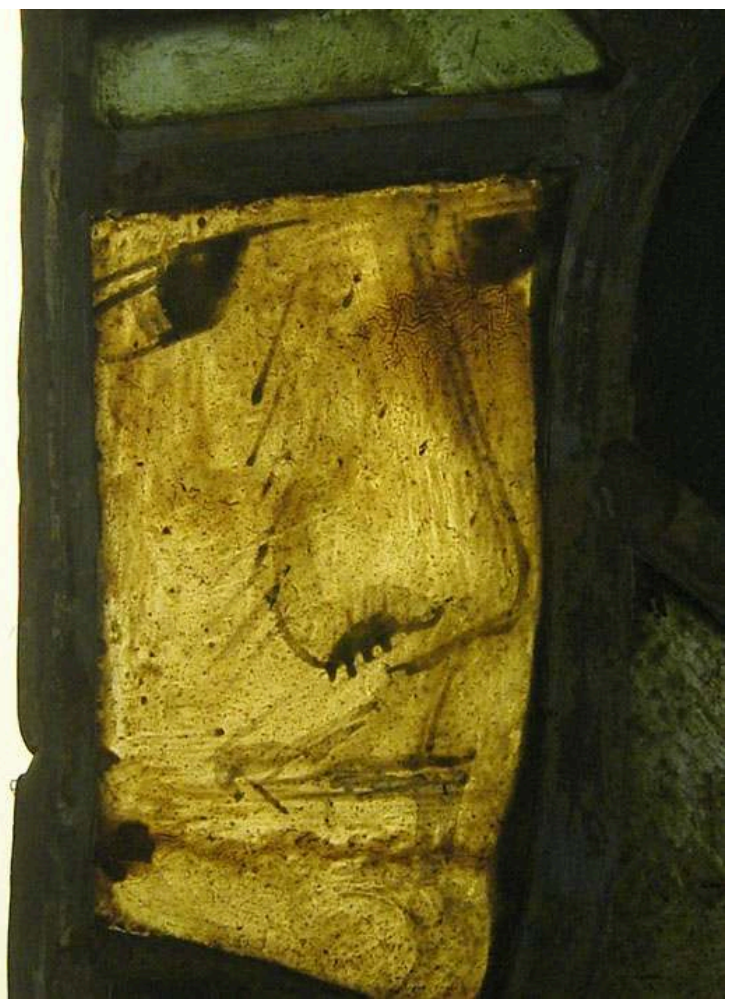

Détail d'un des visages avant restauration.

(c) Michel Blanc-Garin.

31 L'état des douze panneaux était relativement satisfaisant, avec seulement quelques casses limitées à la chevelure de Jeanne d'Arc. Le problème essentiel a été celui de la patine posée à froid. En s'encrassant, elle a généré des effets de réticulations et des épaississements disgracieux, notamment dans les visages (fig. 14). De plus, cette patine avait été retirée des deux panneaux composant la partie supérieure de la Pietà lors d'une restauration antérieure, créant visuellement un désaccord par rapport au reste (fig. 15); la solution adoptée a consisté dans la pose d'une patine réversible à l'extérieur de l'œuvre pour harmoniser l'ensemble. 
Figure 15

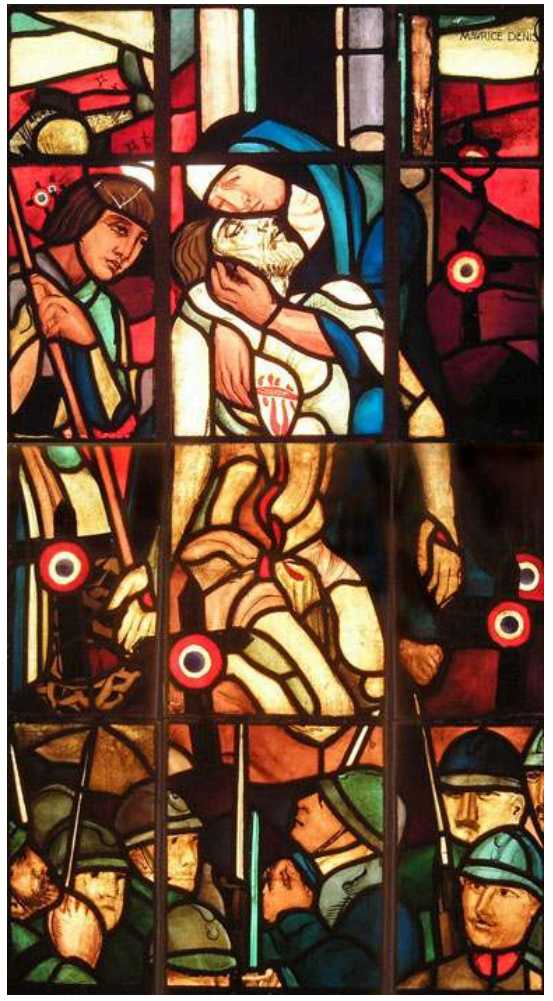

La verrière avant restauration.

(c) Michel Blanc-Garin.

Aujourd'hui, ce vitrail prend place dans la section «Verre» de l'important département Arts décoratifs du musée des Beaux-Arts de Montréal, dont la collection a été récemment publiée avec faste ${ }^{29}$. Il vient compléter le fonds des vitraux de Tiffany (ancienne église Erskine and American, désormais salle Bourgie).

De fait, Maurice Denis occupe une place importante dans l'histoire du vitrail à la fin du $\mathrm{XIX}^{\mathrm{e}}$ siècle et dans la première moitié du Xxe siècle. Admiratif des vitraux du Moyen Âge, il a, dès ses débuts, considéré l'art du vitrail comme un art à part entière, au même titre que la peinture ou le grand décor mural. Il est intervenu dans la réalisation de près de soixante-dix verrières - autant dans le domaine civil que religieux - et a eu, de par ses prises de position en la matière, une influence considérable sur les peintres-verriers de son temps, même sur des ateliers très éloignés de ses conceptions, tels que les frères Mauméjean ${ }^{30}$, dont la verrière de l'église de Pau (fig. 16) n'est pas sans rappeler la maquette de celle pour Saint-Roch de Paris, sans doute vue lors de l'exposition au Pavillon de Marsan en 1920, déjà évoquée. 
Figure 16

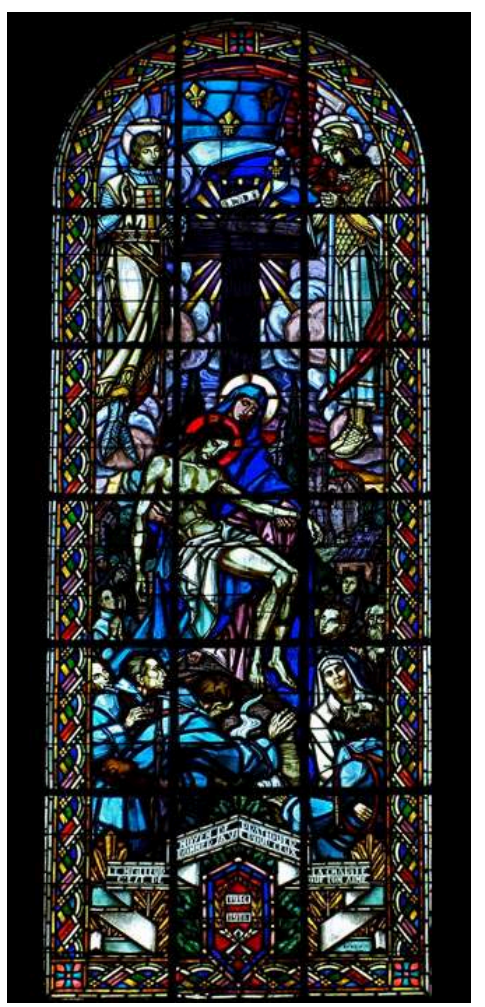

SA Mauméjean frères, La France douloureuse de la guerre, église Notre-Dame, Pau (PyrénéesAtlantiques), 1933.

(c) Benoît Manauté.

Pour compléter la présente étude, il faut remettre ce projet et cette réalisation en perspective dans le corpus des verrières commémoratives, qui fera l'objet d'un chapitre dans l'ouvrage du Corpus des vitraux de Maurice Denis à paraitre aux éditions du Corpus vitrearum (collection Études).

\section{NOTES}

1. - Le catalogue des collections du musée départemental Maurice Denis - Le Prieuré (1e édi. 1980, réédit. 1985) indique : $n^{\circ} 716$ : «Aux morts de la guerre", esquisse pour un vitrail de l'église Saint-Roch de Gagny, gouache sur papier, 87 x $61 \mathrm{~cm}$, daté en b. à g. : « 2e esquisse Avril 1922 », Inv. PMD 976.1.694. L'erreur se retrouve dans : BLIN, Jean-Pierre. « Le vitrail commémoratif de la Grande Guerre. Les catholiques français et le culte du souvenir ». Dans CHALINE, Nadine-Josette. Chrétiens dans la première guerre mondiale. Paris, 1993 (coll. Histoire religieuse de la France), p. 173 (maquette reproduite p. 175, fig. 26). Cette erreur est encore reprise dans Un patrimoine de lumière 1830-2000, Verrières des Hauts-de-Seine, Seine-Saint-Denis, Val-de-Marne, (dir. Laurence de Finance et Dominique Hervier). Paris : Monum. Éditions du patrimoine, 2003, p. 176-177 (coll. Cahiers du Patrimoine, 67). 
2. - Nous avions rédigé un premier article lors de cette découverte : DAVID, Véronique, STAHL, Fabienne. «Aux morts de la guerre de Maurice Denis, projet inédit d'une verrière commémorative pour l'église Saint-Roch ». Aujourd'hui Saint-Roch, n 44, décembre 2005, p. 20 et $n^{\circ} 45$, mars 2006, p. 17. Ce projet était ensuite mis en perspective, dans un second article : CAPPRONNIER, JeanCharles, DAVID, Véronique, STAHL, Fabienne. «Les aménagements commémoratifs dans les églises. L'exemple de George Desvallières, Maurice Denis et Henri Marre ». Le Point riche [bulletin de l'association des Amis de Louis Mazetier], juin 2006. Nous faisions ensuite une notice détaillée, incluant de larges extraits de correspondance, dans : STAHL, Fabienne. Les décorations religieuses de Maurice Denis entre les deux guerres, thèse sous la direction de Jean-Paul Bouillon, Université Blaise Pascal, Clermont-Ferrand, 2008-2009, vol. II, p. 408-413.

3. - Voir le site : http://www.saintrochparis.cef.fr/galerie/.

4. - Documentation du musée départemental Maurice Denis, Saint-Germain-en-Laye, donation de la famille Denis [MMD ensuite], (Ms 8531).

5. - Lettre datée du 12 février 1920 (MMD, Ms 8532).

6. - Lettre de Denis à Peuportier, $1^{\text {er }}$ juillet 1920, archives historiques de l'archevêché de Paris [AHA ensuite].

7. - Il écrit à Denis, le 25 juillet 1920 : "Je suis confus de ne pouvoir encore vous donner une réponse définitive au sujet du beau vitrail dont vous m'avez envoyé le projet. Depuis un mois tous ou presque tous les membres du conseil paroissial sont absents de Paris et je ne puis compter les revoir avant la fin de septembre. Ce n'est qu'à cette époque que je leur soumettrai votre projet avec le prix demandé pour son exécution. Vous voudrez bien excuser ce retard indépendant de ma volonté et agréer l'assurance de ma considération très distinguée » (MMD, Ms 8533).

8. - Lettre de Denis à Peuportier, 25 novembre 1920, AHA : «J'ai reçu de Monsieur Lefébure un mot m'avertissant que la question du vitrail doit rester en suspens jusqu'à ce que vous ayiez pu réunir votre commission. Ayant à participer à l'exposition d'Art Chrétien qui se tiendra au Pavillon de Marsan en décembre prochain, je vous serais reconnaissant de me laisser prendre mon esquisse pour l'y exposer. Je ne vous en priverai que pendant un mois et demi et d'ailleurs, elle ne sera pas très loin de vous : le Musée des Arts décoratifs est sur votre paroisse ».

9. - Musée des Arts décoratifs, Paris, organisée par la Société de Saint-Jean, 14 décembre 1920 31 janvier $1921:$ n$^{\circ} 155$ « Aux morts de la guerre, projet de vitrail pour l'église Saint-Roch ».

10. - Encore inédit, ce projet a été présenté pour la première fois au public dans l'exposition "Maurice Denis au Vésinet ", au musée départemental Maurice Denis à Saint-Germain-en-Laye, du 8 avril au 6 juillet 2014 .

11. - Nous avons relevé, dans l'agenda de Denis de 1921, la mention «S. Roch" aux dates du 30 mai, 9 juin, et 14 juin. L'artiste a donc dû passer à l'église lors de la présentation de sa maquette.

12. - Lettre de P. Rouleau à Maurice Denis, Pâques 1921 (MMD, Ms 9702).

13. - 15 mai 1921 (MMD, Ms 8534). Les AHA conservent le brouillon de cette lettre.

14. - Voir notamment la place du visage de la Vierge par rapport à la barlotière.

15. - Reçu écrit par Denis pour le curé : « [En tête Ateliers d'art Sacré] Reçu de Monsieur le Curé de Saint Roch une esquisse de vitrail par M. Maurice Denis, que je charge mon élève M. Couturier de retirer à la sacristie. 11 mars $1922 »$ (AHA).

16. - Double de la lettre, daté du 17 mai 1922 (AHA).

17. - Lettre de A. Lefébure (en-tête conseil municipal de Paris) au curé, datée du 30 mai 1922 (AHA) : « Voudriez-vous adresser aussi une lettre à Monsieur [Falcere], directeur des Beaux-Arts à l'Hôtel de Ville pour lui demander l'autorisation d'enlever le retable qui est dans la Chapelle des Morts et qui masque votre futur vitrail. Il est prévenu de cette demande et autorisera sûrement l'enlèvement de ce rétable ".

18. - Maurice Denis écrit au chanoine Couget le 6 juin 1922 (cachet de la poste) (AHA) : «Je ne puis m'engager absolument à livrer le vitrail pour novembre car cela dépend en majeure partie 
du verrier qui l'exécutera. Or je ne puis ni lui fournir un carton, ni m'entendre définitivement avec lui tant que la question du retable ne sera pas résolue par la Ville et par les Beaux-Arts. J'ai expliqué à $\mathrm{M}$. Lefébure la marche à suivre et je ne doute pas qu'il ne s'en soit déjà occupé. Aussitôt que vous aurez par lui une réponse officielle, vous voudrez bien m'en avertir. D'ici là, je ferai prendre le dessin définitif que vous avez soumis à vos fabriciens et qui en somme, à part quelques détails, les contente. Je pourrai en commencer l'agrandissement et j'espère que ni la ville, ni les Beaux-Arts ne nous feront trop attendre. Quant aux plaques commémoratives, je vous soumettrai très prochainement un projet. Elles seront prêtes pour novembre. Le vitrail pourra l'être aussi à cette condition que $M$. Lefébure nous donne très vite le moyen de supprimer le retable (ou du moins la partie centrale et envahissante)".

19. - Reçu de Denis au chanoine Couget, 8 juin 1922 (AHA) : «Je prie M. le chanoine Couget de vouloir bien remettre à mon élève Mademoiselle Faure porteur de ce mot le projet de vitrail qu'elle doit lui apporter à Saint-Germain. / Remis le 12 juin 1922 (signé Couget) ».

20. - Denis écrit au chanoine Couget le 13 juin [1922] (AHA) : "J'espère pouvoir débrouillé avec vous la nouvelle complication dont vous me parlez en allant vous rendre visite jeudi 2 heures (après demain) ».

21. - Lettre datée du 14 juin 1922 (AHA), où il écrit : "J'ai lu avec une particulière attention les avis émis par M.M. les Conseillers, en ce qui concerne le monument projeté, pour honorer la mémoire des paroissiens de Saint Roch morts à la guerre. Le côté artistique me paraît très heureusement conçu, et on ne saurait s'en étonner, du moment que M. Maurice Denis est chargé de cette œuvre. Mais le côté financier du projet me semble assez préoccupant. Comme il arrive souvent, le sujet principal du monument entraîne, exige même ici, des dépenses qui n'ont pas été prévues, ou du moins chiffrées assez exactement, et nous ne saurions trop encourager votre Conseil, à établir, d'accord avec vous, avant de rien engager de définitif, un devis minutieux et complet de la dépense qu'entraînerait la réalisation du projet de votre prédécesseur. Nous attendrons résultat de cette première étude pour examiner ensuite la question du déplacement du rétable, qui est conditionnée d'abord par celle des crédits à engager et de vos disponibilités financières. Je vous rappelle qu'en général les dépenses de cette nature sont couvertes par des recettes extraordinaires provenant de la souscription des fidèles et que, dans le cas elles pourraient difficilement être gagées sur les ressources ordinaires de la paroisse ».

22. - Le jeune architecte Jacques Bonnier est chargé de l'aménagement commémoratif; il sollicitera Denis qu'il connaît de Perros-Guirec, qui lui apportera son concours en dépit du retrait du projet (voir les lettres échangées entre les deux hommes).

23. - Lettre du lundi 26 juin 1922, archives Marcel Poncet, Vich (Suisse).

24. - CAPPRONNIER, Jean-Charles, DAVID, Véronique. «Les pavillons des vitraux aux expositions internationales de Paris en 1925 et 1937 ». Revue de l'art, n¹79, 2013-1, p. 41-64.

25. - Archives départementales de Seine-Maritime, fonds Chirol 97 J 53, lettres de Jean HébertStevens à Chirol le 11 juillet 1925. Dans le catalogue de l'Exposition internationale des arts décoratifs et industriels modernes. Pavillon officiel du vitrail. Catalogue, 1925 [s.l], p. 5-6, les œuvres présentées par l'atelier portent les $n^{\circ} 32$ à 42 , le n³9 étant réservé à la Pietà de Maurice Denis.

26. - Deux cocardes sont montées à l'envers. C'est sans doute une erreur du jeune atelier, mais cela donne finalement une certaine profondeur au vitrail selon Michel Blanc-Garin qui a restauré la verrière.

27. - MMD, Ms 9395.

28. - LEDOUX, Florence. L'atelier de vitraux Hébert-Stevens, Rinuy, Bony. Mémoire de DEA sous la direction de Bruno Foucart, professeur d'histoire de l'art contemporain, université de Paris IV, Paris-Sorbonne, U.F.R. d'histoire de l'art et d'archéologie, 1996, fig. 43, hors pagination.

29. - La collection du musée des Beaux-arts de Montréal, Arts décoratifs et design. tome II, (dir. PEPALL, Rosalind et CHARBONNEAU, Diane), 2012, p. 48-49. 
30. - Nous renvoyons le lecteur aux travaux de Benoît Manauté, particulièrement sa thèse de doctorat: "Flambe! Illumine! Embrase!» La place de la manufacture de vitrail et mosaique d'art Mauméjean dans le renouveau des arts industriels franco-espagnols (1862-1957), sous la direction de Dominique Dussol, université de Pau et des pays de l'Adour, 2012.

\section{ABSTRACTS}

After the First World War, monuments designed to commemorate its victims were prevalent in a wide variety of artistic fields, both civil and religious. At the Paris church of Saint-Roch, in 1920, the priest Jean Peuportier commissioned the famous painter and theoretician Maurice Denis (1870-1943) to decorate the chapel of souls in purgatory situated on the south side of the nave. The ambitious project was supposed to comprise a stained glass window and two commemorative plaques giving the names of all the parishioners who had lost their lives during the war but the project was never completed. Research carried out during the preparation of a catalogue raisonné of the work of Maurice Denis has brought attention to the different episodes in this affair and allowed for the preparatory work for the stained glass composition to be correctly identified. Hitherto it was mistakenly associated with work carried out for the Saint-Germain church at Gagny.

$\mathrm{Au}$ lendemain de la Grande guerre, la création de lieux de commémorations se multiplie dans les domaines artistiques les plus divers, civils ou religieux. À l'église Saint-Roch, en 1920, c'est au célèbre peintre et théoricien Maurice Denis (1870-1943) que le curé Jean Peuportier confie l'aménagement de la chapelle des âmes du Purgatoire dans le bas-côté sud de la nef. Cette commande ambitieuse qui comprenait une verrière et deux plaques commémoratives portant les noms des paroissiens de Saint-Roch morts pour la France, n'aboutira jamais. Les recherches menées dans le cadre de la préparation du Catalogue raisonné de l'œuvre de Maurice Denis ont mis en lumière les multiples rebondissements de cette affaire et ont permis de rendre leur véritable identité aux travaux préparatoires de cette verrière, bien connus mais attribués à tort jusqu'ici, à l'église Saint-Germain de Gagny.

\section{INDEX}

Mots-clés: Guerre de 1914-1918, commémoration, Maurice Denis, église Saint-Roch à Paris, Musée des Beaux-arts de Montréal, église Saint-Germain de Gagny, art religieux, Musée départemental Maurice Denis, Poilus, cimetière militaire, peintre verrier

\section{AUTHORS}

\section{FABIENNE STAHL}

attachée de conservation du patrimoine, Catalogue raisonné de l'œuvre de Maurice Denis fab.stahl@free.fr 


\section{VÉRONIQUE DAVID}

ingénieur d'études au Centre André Chastel Veronique.David@paris-sorbonne.fr 